\title{
Preparative Centrifugation
}

National Cancer Institute

\section{Source}

National Cancer Institute. Preparative Centrifugation. NCI Thesaurus. Code C18799.

A type of centrifug ation used to separate cell components. Consists of two basic procedures: differential centrifug ation and density gradient centrifug ation. 